\title{
Trees, set compositions and the twisted descent algebra
}

\author{
Frédéric Patras · Manfred Schocker
}

Received: 14 December 2005 / Accepted: 5 July 2006 /

Published online: 22 July 2006

(C) Springer Science + Business Media, LLC 2006

\begin{abstract}
We first show that increasing trees are in bijection with set compositions, extending simultaneously a recent result on trees due to Tonks and a classical result on increasing binary trees. We then consider algebraic structures on the linear span of set compositions (the twisted descent algebra). Among others, a number of enveloping algebra structures are introduced and studied in detail. For example, it is shown that the linear span of trees carries an enveloping algebra structure and embeds as such in an enveloping algebra of increasing trees. All our constructions arise naturally from the general theory of twisted Hopf algebras.
\end{abstract}

Keywords Increasing tree $\cdot$ Set composition $\cdot$ Descent algebra $\cdot$ Twisted Hopf algebra

\section{Introduction}

The direct sum of the Solomon-Tits algebras of type $A_{n}$, or twisted descent algebra, has been shown in [16] to carry a rich algebraic structure which extends and generalizes the structures on the classical descent algebra (the direct sum of the Solomon algebras of type $A_{n}$ ). From a combinatorial point of view, moving from the classical to the twisted descent algebra means moving from the combinatorics of compositions (sequences of integers) to the combinatorics of set compositions (sequences of mutually disjoint sets).

F. Patras $(\bowtie)$

CNRS, UMR 6621, Parc Valrose, 06108 Nice cedex 2, France

e-mail:patras@math.unice.fr

M. Schocker

Department of Mathematics, University of Wales Swansea, Singleton Park, Swansea SA2 8PP, UK e-mail: m.schocker@swansea.ac.uk 
The purpose of the present article is to pursue further the study of the algebraic structures associated to set compositions, or twisted descents, and related objects. Fields of application of the theory include, among others, the geometry of Coxeter complexes of type $A_{n}$, the internal structure of twisted Hopf algebras, Markov chains associated to hyperplane arrangements, and Barratt's twisted Lie algebra structures in homotopy theory. We refer to [16] for a survey of the history of the subject, and further details on its various fields of application.

We first show, in Section 2, that the natural basis of the Solomon-Tits algebra of type $A_{n}$ (or, equivalently, the set of faces of the hyperplane arrangement of type $A_{n}$, the set of set compositions of $\{1, \ldots, n\}$, or the set of cosets of standard parabolic subgroups in the symmetric group $S_{n}$ ) is in bijection with the set of increasing planar rooted trees with $n$ branchings. The result is new, to our best knowledge, although it appears to be a very natural extension of the classical bijection between permutations and increasing planar binary trees (see, for example, [17, 19] and the appendix of [9]), and of the mapping from set compositions to planar rooted trees introduced in [21] and further studied in [5]. This provides another link between combinatorial structures, Hopf algebras and trees. This domain has received a considerable attention recently, due in particular to the discovery of its role in the understanding of high energy physics through the seminal work of Connes and Kreimer on Feynman graphs and Zimmermann's renormalization formula [6,7]. Other approaches and other problems have also enlightened the explanatory power of this link. These influential contributions include Chapoton's work on Hopf algebras, trees and the geometry of Coxeter complexes [5], Loday's and Ronco's work on planar binary trees and operads [10], and the work of Brouder-Frabetti on planar binary trees and QED [4].

Set compositions of finite sets of positive integers are also in 1-1 correspondence with monomials in non-commuting variables $x_{1}, x_{2}, \ldots$ This connects our work with the theory of word quasi-symmetric functions, WQsym, and the theory of quasi-symmetric functions in non-commuting variables, NCQsym, recently introduced by Hivert et al. (see, for example, [12]) and Bergeron et al. (see, for example, [2, 3]), respectively.

In Sections 3 and 4, we revisit the twisted Hopf algebra structure on the twisted descent algebra introduced in [16] and start to analyze the algebraic implications of our combinatorial result. We study in detail the two Hopf algebra structures on increasing planar rooted trees which are induced by this twisted Hopf algebra, by means of the symmetrisation and cosymmetrisation processes of $[15,20]$.

One of these Hopf algebras is neither commutative nor cocommutative. This Hopf algebra turns out to be dual to the algebra NCQsym, thereby revealing a different approach to the algebra of quasi-symmetric functions in non-commuting variables. This observation has a striking consequence. Namely, the classical triple at the heart of Lie theory (Solomon algebra of type $A_{n}$; descent algebra (the direct sum of Solomon algebras); quasi-symmetric functions) lifts to the world of twisted objects as a triple (Solomon-Tits algebra of type $A_{n}$; twisted descent algebra; quasi-symmetric functions in non-commuting variables). The link between the first two objects was investigated in detail in [16], whereas the duality properties between the twisted descent algebra and the algebra of quasi-symmetric functions in non-commuting variables are a byproduct of our considerations in Section 3 of the present article. This emphasizes, once again, the conclusion drawn from $[15,16,18]$ that the twisted descent algebra 
is the "natural framework" to lift classical algebraic and combinatorial structures (compositions, descents, shuffles, free Lie algebras, and so on) to the enriched setting of set compositions, tensor species, Barratt's free twisted Lie algebras, and so on.

The other Hopf algebra structure provides the twisted descent algebra (or, equivalently, the linear span of increasing planar rooted trees), with the structure of an enveloping algebra. This is the Hopf algebra we will be mainly interested in, in view of its rich algebraic and combinatorial structure. We show, for example, that the twisted descent algebra is a free associative algebra with generators in bijection with so-called balanced increasing rooted trees, and furthermore, naturally, the enveloping algebra of a free Lie algebra that we describe explicitly.

In final Section 5, we study Hopf subalgebras of the twisted descent algebra. The relationships of the noncommutative noncocommutative Hopf algebra structure on set compositions to its most remarkable Hopf subalgebras appear quite simple and natural. For example, whereas Chapoton's Hopf algebra [5] is related to the MalvenutoReutenauer Hopf algebra by means of a sub-quotient construction, we can show that the natural embedding of the free twisted associative algebra on one generator into the twisted descent algebra implies that the (noncommutative noncocommutative) Hopf algebra we consider contains Malvenuto-Reutenauer's (and therefore all its Hopf subalgebras) as proper Hopf subalgebras.

However, our main concern is, once again, the other structure, that is, the cocommutative case. We show that the direct sum of the symmetric group algebras, when provided with the enveloping algebra structure introduced in [15, Section 6], embeds as an enveloping algebra in the twisted descent algebra. We recover in particular, as a corollary of our results on the twisted descent algebra, Theorem 21 in [15], stating that the Lie algebra of primitive elements in this direct sum is a free Lie algebra.

Finally, we also introduce enveloping algebra structures on planar trees and planar binary trees which seem to be new. We show that these enveloping algebras embed into the enveloping algebra of increasing planar rooted trees as well.

\section{Set compositions and planar rooted trees}

We give here a 1-1 correspondence between set compositions and increasing planar rooted trees which extends a construction of [21] (see also [5]). Our bijection also extends the classical correspondence between increasing planar binary rooted trees and permutations.

Let $n$ be a non-negative integer and set $[n]:=\{1,2, \ldots, n\}$. A set composition of [ $n$ ] of length $k$ is a $k$-tuple $P=\left(P_{1}, \ldots, P_{k}\right)$ of mutually disjoint non-empty subsets $P_{1}, \ldots, P_{k}$ of $[n]$ such that $P_{1} \cup \ldots \cup P_{k}=[n]$. There is an obvious $1-1$ correspondence between surjective maps $\varphi:[n] \rightarrow[k]$ and set compositions $P$ of length $k$ of $[n]$ which assigns to any such $\varphi$ the $k$-tuple $P=\left(\varphi^{-1}(1), \varphi^{-1}(2), \ldots, \varphi^{-1}(k)\right)$. For example, if $n=3, k=2$ and $\varphi(1)=1=\varphi(3), \varphi(2)=2$, then $P=(13,2)$. Note that we dropped several commas and curly brackets in $P$.

In what follows, a (planar, rooted) tree is a finite planar non-empty oriented connected graph $T$ without loops such that any vertex of $T$ has at least two incoming edges and exactly one outgoing edge. In illustrations, the root appears at the bottom, the leaves appear at the top, and the orientation is dropped with the understanding 


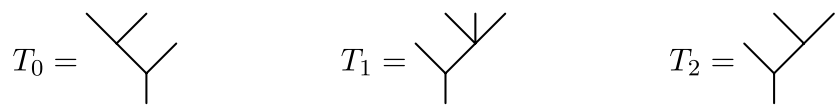

Fig. 1 Three trees

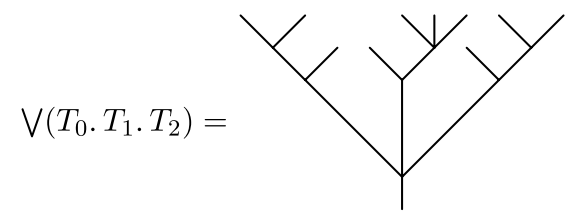

Fig. 2 The wedge of three trees

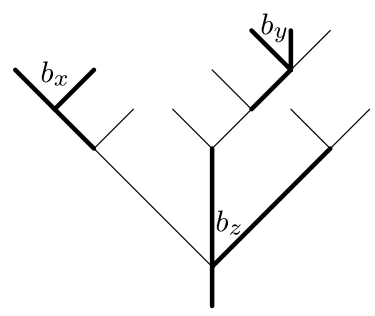

Fig. 3 Three branchings

that all edges are oriented from top to bottom. Three trees $T_{0}, T_{1}, T_{2}$ with two vertices and, respectively, three, four and three leaves are displayed in Fig. 1. The trivial tree without vertex will be denoted by $\varepsilon$.

If $m \geq 1$, then the wedge of $m+1$ trees $T_{0}, T_{1}, \ldots, T_{m}$ is obtained by joining the roots of $T_{0}, \ldots, T_{m}$ to a new vertex and creating a new root. It is denoted by $\bigvee\left(T_{0}, T_{1}, \ldots, T_{m}\right)$. For example, the wedge of the trees $T_{0}, T_{1}, T_{2}$ in Fig. 1 is given in Fig. 2. Any tree $T \neq \varepsilon$ can be written uniquely as the wedge $\bigvee\left(T_{0}, T_{1}, \ldots, T_{m}\right)$ of certain subtrees of $T$. We define the $m$-corolla $C_{m}$ by $C_{m}=\bigvee(\underbrace{\varepsilon, \varepsilon, \ldots, \varepsilon}_{m})$. Hence $C_{m}$ is the unique tree with one vertex and $m$ leaves. For example, $C_{4}=\Psi$.

Tonks [21] and Chapoton [5] studied a surjective map which assigns to each set composition $P$ a tree $T$. We will consider additional structure on trees which will allow us to turn this surjection into a bijection.

A branching $b$ of a tree $T$ is a subgraph of $T$ isomorphic to $C_{2}=Y$. Three branchings $b_{x}, b_{y}, b_{z}$ are indicated in Fig. 3 .

We denote the set of all branchings of $T$ by $B(T)$, and its cardinality by $b(T)$. The set $B(T)$ admits a "left-to-right" order $\preceq$ in a natural way: if $T$ is the $m+1$-corolla $C_{m+1}$ for some $m \geq 0$, then $T$ has $m$ branchings $b_{1}, b_{2}, \ldots, b_{m}$ (labelled from left to right) and we define $b_{1} \preceq b_{2} \preceq \cdots \preceq b_{m}$; see Fig. 4. If, more generally, $T$ is the wedge of (possibly non-empty) trees $T_{0}, \ldots, T_{m}$, we proceed by induction and extend the orders on $B\left(T_{0}\right), \ldots, B\left(T_{m}\right)$ to an order on $B(T)$ by setting

$$
b_{i} \prec b \prec b_{i+1}
$$




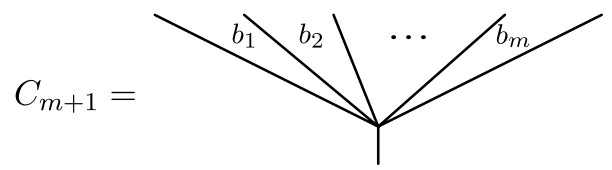

Fig. 4 The branchings of the $m+1$-corolla

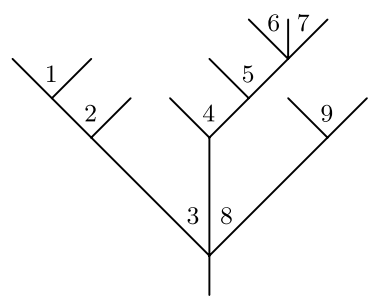

Fig. 5 Natural labelling of the branchings

for all $i \in\{0,1, \ldots, m\}$ and $b \in B\left(T_{i}\right)$, where $T_{i}$ is embedded in $T$. (The term $b_{i}$ on the left, respectively, $b_{i+1}$ on the right, does not appear when $i=0$, respectively, when $i=m$.) For example, for the branchings indicated in Fig. 3, we obtain $b_{x} \preceq b_{y} \preceq b_{z}$.

The order $\preceq$ induces a natural labelling of the branchings of $T$, namely the orderpreserving map $([n], \leq) \rightarrow(B(T), \preceq)$, where $n=b(T)$ and $\leq$ denotes the usual order on $[n]$. The natural labelling of the tree in Fig. 3 is illustrated in Fig. 5.

A root branching of $T$ is a branching $b \in B(T)$ which contains the root. The tree in Fig. 5, for example, has two root branchings, with natural labels 3 and 8. With $T=\bigvee\left(T_{0}, \ldots, T_{m}\right)$ as above, the natural label of a root branching $b_{i}$ is

$$
x_{i}=i+\sum_{j=0}^{i-1} b\left(T_{j}\right)
$$

for all $i \in[m]$. Furthermore, if $i \in[m] \cup\{0\}, \tilde{b} \in B\left(T_{i}\right)$ has natural label $j$ in $T_{i}$ and $b$ is the branching of $T$ corresponding to $\tilde{b}$ (via the embedding of $T_{i}$ in $T$ ), then $b$ has natural label $x_{i}+j$ in $T$, where $x_{0}:=0$.

A level function on a tree $T$ is a surjective map $\lambda: V \rightarrow X$ such that $\lambda$ is strictly increasing along each path connecting a leaf of $T$ with the root of $T$. Here $V$ is the set of vertices of $T$ and $X$ is a totally ordered set. Such a level function is said to be standard if $X=[k]$ (with the usual order), for some non-negative integer $k$. A tree provided with a standard level function is an increasing tree.

Three standard level functions are illustrated in Fig. 6. It is easy to see that there are no other standard level functions on this tree.

Suppose $X$ has order $k$ and $\iota: X \rightarrow[k]$ is the order-preserving bijection. It is clear that any level function $\lambda: V \rightarrow X$ on $T$ yields the standard level function $\iota \circ \lambda$ on $T$. We refer to this standard level function as the standardization of $\lambda$. It will be
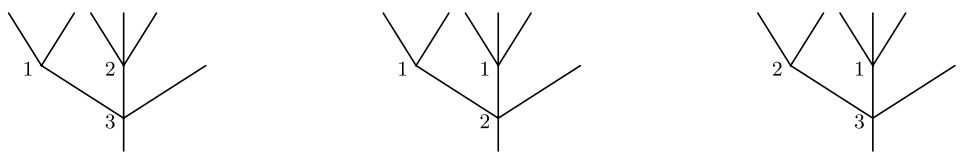

Fig. 6 Standard level functions 


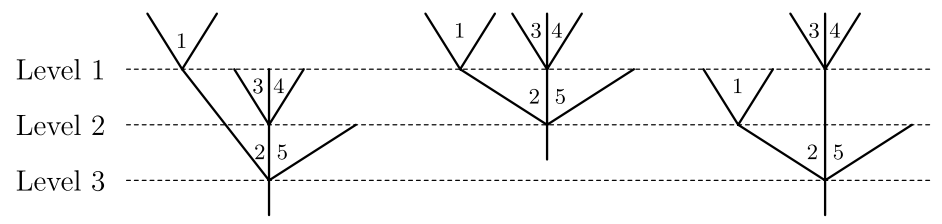

Fig. 7 Increasing trees

advantageous at a later stage to consider arbitrary ordered sets $X$ in the definition of a level function.

When illustrating an increasing tree, it is more convenient to draw each vertex $v$ at the level $\lambda(v)$ (where levels increase from top to bottom) rather than to label $v$ by $\lambda(v)$; see Fig. 7. (We have added here the natural labels of the branchings in each case for purposes which will be clear later.)

Some geometrical properties of increasing trees can be described in terms of natural labels and levels. For example, the vertices of two branchings $b$ and $b^{\prime}$ of an increasing tree $T$ with natural labels $i<i^{\prime}$ at levels $l<l^{\prime}$ (or $l>l^{\prime}$, respectively) belong to a common path joining a leaf of $T$ to the root of $T$ if and only if the vertices of all the branchings $d$ with labels between $i$ and $i^{\prime}$ have levels (strictly) greater than $l$ (or $l^{\prime}$, respectively).

If $\lambda: V \rightarrow X$ is a level function on $T$ and $b$ is a branching of $T$ with vertex $v$, we assign to $b$ the level $\lambda(v)$. Let $n=b(T)$, and suppose that $X=[k]$ for some non-negative integer $k$. Then the composite of the natural labelling of $B(T)$ with the standard level function $\lambda$ (extended to $B(T)$ in the way described) yields a surjective map $[n] \rightarrow[k]$ or, equivalently, a set composition

$$
\sigma(T, \lambda)=P=\left(P_{1}, \ldots, P_{k}\right)
$$

More explicitly, $P_{i}$ consists of the natural labels of all branchings of $T$ at level $i$, for all $i \in[k]$. The set compositions arising in this way from the increasing trees given in Fig. 7 are, respectively, $(1,34,25),(134,25)$ and $(34,1,25)$.

Theorem 2.1. The map $(T, \lambda) \mapsto \sigma(T, \lambda)$ is a bijection from the set of all increasing trees with $n$ branchings onto the set of all set compositions of $[n]$.

By restriction, we obtain a correspondence between increasing binary trees and permutations, identified with set compositions of $[n]$ of length $n$. Note that, in this correspondence, a permutation $\pi$ is associated to the binary tree which is classically associated to $\pi^{-1}$; see $[17,19]$ and the appendix of [9].

In order to prove the theorem, we give an inductive description of the inverse map $\tau$ of $\sigma$. For this purpose, it is convenient to identify a standard level function $\lambda: B(T) \rightarrow$ $[k]$ on a tree $T$ with $n$ branchings with the corresponding map $\varphi:[n] \rightarrow[k]$, by means of the natural labelling of the branchings.

Let $P=\left(P_{1}, \ldots, P_{k}\right)$ be any set composition of $[n]$, and denote the corresponding surjective map from $[n]$ to $[k]$ by $\varphi$. Define $T_{1}$ to be the 2-corolla with level function $\lambda_{1}=\left.\varphi\right|_{[1]}$, the restriction of $\varphi$ to [1]. Let $i \in[n-1]$, and assume inductively that a Springer 


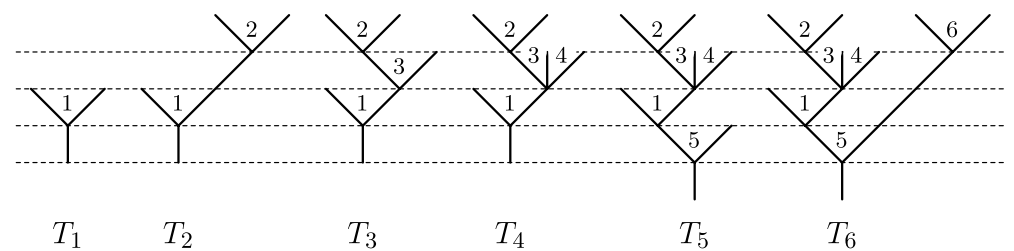

Fig. 8 Construction of the increasing tree corresponding to $(26,34,1,5)$

tree $T_{i}$ has been constructed with level function $\lambda_{i}=\left.\varphi\right|_{[i]}$. Then define $\left(T_{i+1}, \lambda_{i+1}\right)$ to be the (unique) increasing tree with $i+1$ branchings obtained by adding to $\left(T_{i}, \lambda_{i}\right)$ a branching on the right at level $\varphi(i+1)$. After $n$ steps, we arrive at a tree $T_{n}$ with $n$ branchings and standard level function $\lambda_{n}=\varphi$. We set

$$
\tau(P):=\left(T_{n}, \lambda_{n}\right)
$$

It is immediate from the definitions that $\tau$ is a left and a right inverse of $\sigma$. This proves the theorem.

The construction is best understood through an example. The case where $P=$ $(26,34,1,5)$ is illustrated in Fig. 8.

The composite of $\tau$ with the forgetful map from increasing trees to trees can be shown to agree with Chapoton's map $\Psi$ (see [5, pp. 267]), although their definitions might look different at first sight. The proof is left to the reader.

To conclude this section, we observe that the construction of $\tau(P)$ applies, more generally, to an arbitrary surjective map $\varphi$ from a finite ordered set $A$ onto an ordered set $X$ (instead of the surjection $\varphi:[n] \rightarrow[k]$ corresponding to $P$ ). As a result, we obtain a tree with branchings labeled by $A$ and levels drawn from $X$. In particular, we can assign an increasing tree $\tau(Q)$ with labels in an ordered set $A$ to a set composition $Q$ of length $k$ of an arbitrary finite ordered set $A$ (since such a set composition corresponds to a surjective map $\varphi: A \rightarrow[k])$.

If $P=\left(P_{1}, \ldots, P_{k}\right)$ is a set composition of $[n]$ and $A$ is a subset of $[n]$, then $Q:=$ $\left(P_{1} \cap A, \ldots, P_{k} \cap A\right)$ is a set composition of $A$, where the upper index \# indicates that empty sets are deleted. In geometric terms, the increasing tree $\tau(Q)$ is then obtained from the increasing tree $(T, \lambda):=\tau(P)$ by "contracting" in a certain way all branchings of $T$ with natural labels not contained in $A$ (and keeping the levels of branchings with labels in $A$ ). Accordingly, $\tau(Q)$ is called the contraction of $(T, \lambda)$ relative to $A$, or $A$ contraction of $(T, \lambda)$. An example is displayed in Fig. 9, where $(T, \lambda)$ is the increasing tree corresponding to the set composition $P=(26,34,1,5)$ and $A=\{1,2,4,6\}$. The notion of contraction will play a vital role in our constructions of coproducts on the linear span of increasing trees in the sections that follow.

\section{The twisted descent algebra as a Hopf algebra}

Let $\mathbb{Z}$ and $\mathbb{N}$ denote the sets of all integers and of all positive integers, respectively, and set $\mathbb{N}_{0}:=\mathbb{N} \cup\{0\}$. The free twisted descent algebra $\mathcal{T}$ has $\mathbb{Z}$-linear basis the set 

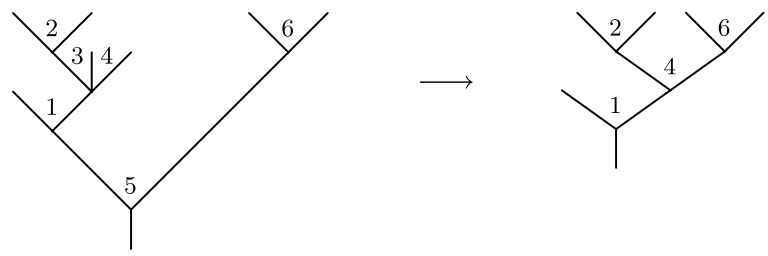

Fig. 9 The contraction of $(26,34,1,5)$ relative to $A=\{1,2,4,6\}$

of all set compositions of finite subsets of $\mathbb{N}$. Equivalently, by Theorem 2.1, we can take the set of all increasing trees (with natural labels drawn from $\mathbb{N}$ ) as a basis of $\mathcal{T}$.

The algebra $\mathcal{T}$ is a twisted Hopf algebra. It was shown in [15], for arbitrary twisted Hopf algebras $(H, *, \delta)$, that the twisted product $*$ induces two (ordinary) products on $H$ and that the twisted coproduct $\delta$ induces two (ordinary) coproducts on $H$. These turn $H$ into an ordinary Hopf algebra in two different ways.

In this section, we will make explicit these Hopf algebra structures on $\mathcal{T}$ and thereby explore the algebraic implications of the definitions given in [16] for the study of set compositions and increasing trees. In particular, we will show that one of our constructions recovers the graded dual of the algebra of quasi-symmetric functions in non-commuting variables considered in [3] (see Lemmas 3.4 and 3.7).

For notational brevity, we refer to the free twisted descent algebra of [16] simply as "the twisted descent algebra". All graded vector spaces considered here are connected: their degree 0 component is naturally isomorphic to the ground ring. Hence the two notions of bialgebra and of Hopf algebra coincide on these spaces, and there is no need to specify the antipode.

Let us recall some definitions. More details and references on twisted algebraic structures can be found in $[15,16,18]$. A tensor species is a functor from the category of finite sets and set isomorphisms Fin to the category Mod of vector spaces over a field or modules over a commutative ring. Unless otherwise specified, we will work over $\mathbb{Z}$, so that Mod is the category of abelian groups. For convenience, we will also assume that the finite sets we consider (and therefore the objects in Fin) are subsets of $\mathbb{N}$.

The category Sp of tensor species is a linear symmetric monoidal category for the tensor product defined by:

$$
(F \otimes G)(S):=\bigoplus_{T \amalg U=S} F(T) \otimes G(U)
$$

for all $F, G \in \mathrm{Sp}, S \in$ Fin. Here $T \amalg U=S$ means that $S$ is the disjoint union of $T$ and $U$.

Let Comp $_{S}$ denote the set of set compositions of $S$, for all $S \in$ Fin. As a tensor species, the twisted descent algebra $\mathcal{T}$ can be identified with the linearization of the set composition functor:

$$
\mathcal{T}(S):=\mathbb{Z}\left[\mathrm{Comp}_{S}\right]
$$

We also write $\mathcal{T}_{S}$ instead of $\mathcal{T}(S)$.

The twisted descent algebra carries two products: the internal or composition product $\circ$, and the external or convolution product $*$. It also carries a coproduct $\delta$. All 悬 Springer 
three structures are induced by the natural action of set compositions on twisted Hopf algebras [16]. They are defined as follows.

Definition 3.1. The Fin-graded components $\mathcal{T}_{S}$ of $\mathcal{T}$ are associative unital algebras for the composition product $\circ$, defined by:

$$
\begin{aligned}
& \left(P_{1}, \ldots, P_{k}\right) \circ\left(Q_{1}, \ldots, Q_{l}\right) \\
& \quad:=\left(P_{1} \cap Q_{1}, \ldots, P_{1} \cap Q_{l}, \ldots, P_{k} \cap Q_{1}, \ldots, P_{k} \cap Q_{l}\right)^{\#}
\end{aligned}
$$

for all $\left(P_{1}, \ldots, P_{k}\right),\left(Q_{1}, \ldots, Q_{l}\right) \in$ Comp $_{S}$. As in Section 2, the upper index \# indicates that empty sets are deleted.

The algebra $\left(\mathcal{T}_{[n]}, \circ\right)$ is now widely referred to as the "Solomon-Tits algebra", a terminology introduced in the preprint version of [16]. The connections between Tits's seminal ideas (which ultimately led to the definition of the product $\circ$ ) and graded Hopf algebraic structures were also first emphasized in [16] and have since been subject to increasing interest; see, for example, [3] and [12]. We do not study here the internal product and refer to [18] for detailed structure results on the algebra $\left(\mathcal{T}_{S}, \circ\right)$.

Recall that a twisted associative algebra is an associative algebra in the symmetric monoidal category of tensor species. Twisted versions of coassociative coalgebra, bialgebra, and so on, are defined in the same way.

Definition 3.2. The functor $\mathcal{T}$ is provided with the structure of an associative unital twisted algebra by the convolution product $*$, defined by:

$$
\left(P_{1}, \ldots, P_{k}\right) *\left(Q_{1}, \ldots, Q_{l}\right):=\left(P_{1}, \ldots, P_{k}, Q_{1}, \ldots, Q_{l}\right)
$$

for all $S, T \in$ Fin with $S \cap T=\emptyset$ and all $\left(P_{1}, \ldots, P_{k}\right) \in \operatorname{Comp}_{S},\left(Q_{1}, \ldots, Q_{l}\right) \in$ Comp $_{T}$. The identity element in $(\mathcal{T}, *)$ is the empty tuple $\emptyset$.

The functor $\mathcal{T}$ is also provided with the structure of a coassociative cocommutative counital twisted coalgebra by the coproduct $\delta$, defined by:

$$
\delta\left(P_{1}, \ldots, P_{k}\right):=\sum_{Q_{i} \amalg R_{i}=P_{i}}\left(Q_{1}, \ldots, Q_{k}\right)^{\#} \otimes\left(R_{1}, \ldots, R_{k}\right)^{\#}
$$

for all $S \in$ Fin, $\left(P_{1}, \ldots, P_{k}\right) \in$ Comp $_{S}$. For example,

$$
\begin{aligned}
\delta(14,7)= & (14,7) \otimes \emptyset+(14) \otimes(7)+(1,7) \otimes(4)+(4,7) \otimes(1) \\
& +(1) \otimes(4,7)+(4) \otimes(1,7)+(7) \otimes(14)+\emptyset \otimes(14,7) .
\end{aligned}
$$

Note that $\delta(P) \in(\mathcal{T} \otimes \mathcal{T})[S]=\bigoplus_{A \amalg B=S} \mathcal{T}_{A} \otimes \mathcal{T}_{B}$ for all $P \in$ Comp $_{S}$. We write $\delta_{A, B}$ for the component of the image of $\delta$ in $\mathcal{T}_{A} \otimes \mathcal{T}_{B}$, so that, for example, $\delta_{\{1,7\},\{4\}}(14,7)=(1,7) \otimes(4)$.

The following is an immediate consequence of [16]. 
Proposition 3.3. The triple $(\mathcal{T}, *, \delta)$ is a cocommutative twisted Hopf algebra.

Whenever $S, T \in$ Fin have the same cardinality $n$, the unique order-preserving bijection $S \rightarrow T$ induces a linear isomorphism is $s_{S, T}: \mathcal{T}_{S} \rightarrow \mathcal{T}_{T}$ in an obvious way. If, in particular, $T=[n]$, then we write $\mathcal{T}_{n}:=\mathcal{T}_{[n]}$ and is ${ }_{S}$ for the isomorphism from $\mathcal{T}_{S}$ onto $\mathcal{T}_{n}$.

We will now use the isomorphisms is ${ }_{S, T}$ to describe the algebra and coalgebra structures on the twisted descent algebra which arise from the general constructions of [15]. For this purpose, let us introduce the graded vector space

$$
\mathcal{T}_{\bullet}:=\bigoplus_{n \in \mathbb{N}_{0}} \mathcal{T}_{n}
$$

Lemma 3.4. The vector space $\mathcal{T}_{\bullet}$ is a graded coassociative counital coalgebra with respect to the restricted coproduct $\bar{\delta}$, defined on the nth component by

$$
\bar{\delta}:=\bigoplus_{p+q=n}\left(\mathcal{T}_{p} \otimes \text { is }_{p+[q]}\right) \circ \delta_{[p], p+[q]}
$$

where we write $\mathcal{T}_{p}$ for the identity on $\mathcal{T}_{p}$ and $p+[q]$ for $\{p+1, \ldots, p+q\}$.

This is a direct consequence of the definition of a twisted coalgebra.

For any $A \subseteq[n]$ and any set composition $P=\left(P_{1}, \ldots, P_{k}\right)$ of $[n]$, we write

$$
\left.P\right|_{A}:=\text { is }_{A}\left(\left(P_{1} \cap A, \ldots, P_{k} \cap A\right)^{\#}\right)
$$

so that, for example, $\left.(35,62,1,47)\right|_{\{1,3,5,7\}}=\operatorname{is}_{\{1,3,5,7\}}\left((35, \emptyset, 1,7)^{\#}\right)=(23,1,4)$. Then, in particular, we have

$$
\bar{\delta}(P)=\left.\left.\sum_{p+q=n} P\right|_{[p]} \otimes P\right|_{p+[q]}
$$

for all $n \in \mathbb{N}_{0}, P \in \operatorname{Comp}_{[n]}$. A comparison with [3, Eq. (21)] now shows that the linear map $W:\left(\mathcal{T}_{\bullet}, \bar{\delta}\right) \rightarrow\left(\right.$ NCQsym* $\left.{ }^{*} \Delta^{*}\right)$, defined by $P \mapsto W_{P}$, is an isomorphism of coalgebras.

The restricted coproduct translates naturally into the language of trees, by means of Theorem 2.1. If $p+q=n$, then the $\operatorname{map}\left(\mathcal{T}_{p} \otimes\right.$ is $\left._{p+[q]}\right) \circ \delta_{[p], p+[q]}$ sends an increasing tree $T$ to $T_{1} \otimes T_{2}$, where $T_{1}$ is the [p]-contraction of $T$ and $T_{2}$ is the (standardization of) the $(p+[q])$-contraction of $T$, as defined at the end of Section 2. Consider, for example, the tree $T$ displayed in Fig. 5, interpreted as an increasing tree with branchings 6 and 7 at level 1 , branchings 1 and 5 at level 2, and so on. The $(5,4)$ component of $\bar{\delta}(T)$ is given in Fig. 10 .

Lemma 3.5. The vector space $\mathcal{T}_{\bullet}$ is a graded coassociative cocommutative counital coalgebra with respect to the cosymmetrized coproduct $\hat{\delta}$, defined on the nth 包 Springer 


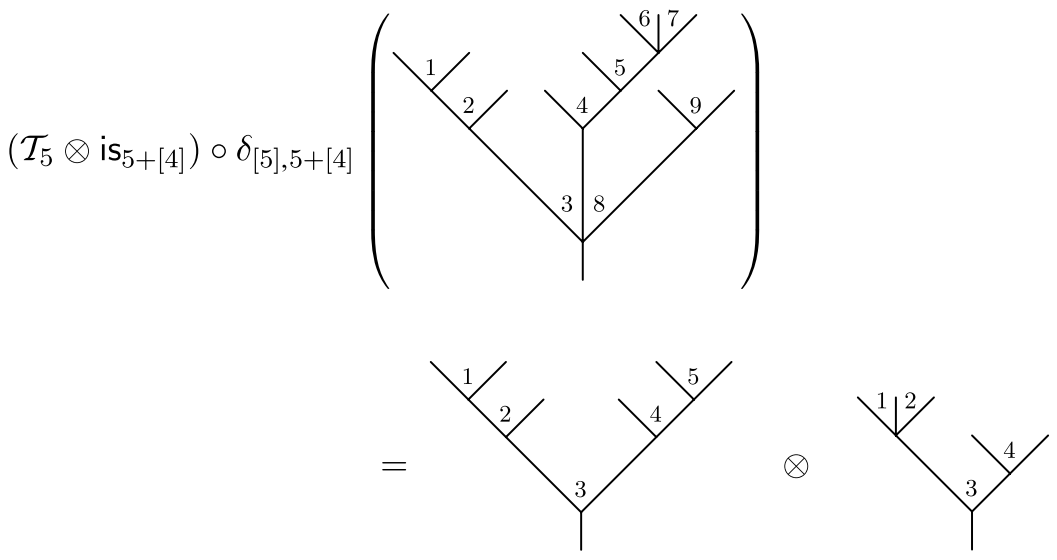

Fig. 10 The $(5,4)$ component of the restricted coproduct $\bar{\delta}$

component by

$$
\hat{\delta}:=\bigoplus_{A \amalg B=[n]}\left(\text { is }_{A} \otimes \text { is }_{B}\right) \circ \delta_{A, B} .
$$

This follows from [15, pp. 207]. Equivalently, we have

$$
\hat{\delta}(P)=\left.\left.\sum_{A \amalg B} P\right|_{A} \otimes P\right|_{B}
$$

for all $n \in \mathbb{N}_{0}, P \in \operatorname{Comp}_{[n]}$.

The cosymmetrized coproduct on increasing trees can also be described in terms of the contraction process introduced in Section 2. If $T$ is an increasing tree with $n$ branchings and $A \amalg B=[n]$, then the $(A, B)$ component of $\hat{\delta}(T)$ reads $T_{A} \otimes T_{B}$, where $T_{A}$ and $T_{B}$ are the contractions of $T$ relative to $A$ and $B$, respectively.

For example, if $T$ is the increasing tree displayed in Fig. 5 again and $A=\{1,2,5,8\}$, $B=\{3,4,6,7,9\}$, then the $(A, B)$ component of $\hat{\delta}(T)$ is displayed in Fig. 11 .

Lemma 3.6. The vector space $\mathcal{T}_{\bullet}$ is a graded associative unital algebra with respect to the restricted product $\bar{*}$, defined on $\mathcal{T}_{p} \otimes \mathcal{T}_{q}$ by

$$
\bar{*}:=* \circ\left(\mathcal{T}_{p} \otimes \mathrm{is}_{[q], p+[q]}\right)
$$

for all $p, q \in \mathbb{N}_{0}$.

This is again a direct consequence of the definition of a twisted algebra. In terms of trees, the restricted product is obtained by grafting an increasing tree $T_{1}$ with $p$ branchings on the left-most leaf of an increasing tree $T_{2}$ with $q$ branchings, resulting in a new tree $T$ with $p+q$ branchings. The level function on $T$ is obtained by keeping 


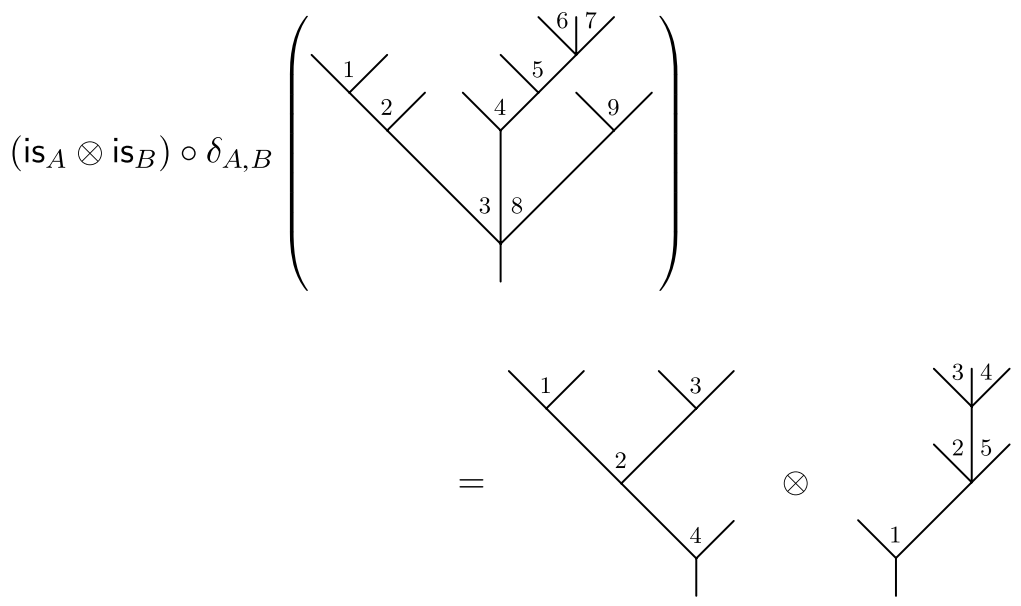

Fig. 11 The $(\{1,2,5,8\},\{3,4,6,7,9\})$ component of the cosymmetrized coproduct $\hat{\delta}$

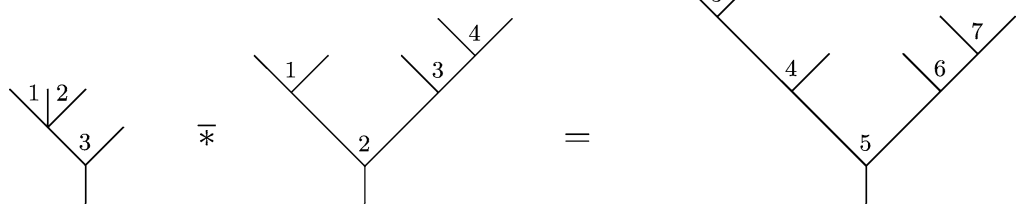

Fig. 12 The restricted product $\bar{*}$

the levels of $T_{1}$ (viewed now as a subtree of $T$ ) and adding the root level $m$ of $T_{1}$ to all levels of $T_{2}$ (viewed also as a subtree of $T$ ). An example is given in Fig. 12.

Lemma 3.7. The vector space $\mathcal{T}_{\bullet}$ is a graded associative unital algebra with respect to symmetrized product $\hat{*}$, defined on $\mathcal{T}_{p} \otimes \mathcal{T}_{q}$ by

$$
\hat{*}:=\sum_{A \amalg B=[p+q],|A|=p,|B|=q} * \circ\left(\mathrm{is}_{[p], A} \otimes \mathrm{is}_{[q], B}\right)
$$

for all $p, q \in \mathbb{N}_{0}$.

This construction is dual to the construction of $\hat{\delta}$ (see [15, pp. 212]). A comparison with [3, Eq. (20)] shows that the map $W$ considered after Lemma 3.4 is also an isomorphism of algebras from $\left(\mathcal{T}_{\bullet}, \hat{*}\right)$ onto NCQsym*.

The symmetrized product is slightly more difficult to describe in terms of increasing trees and should be thought of as the right notion of "shuffle product" for increasing trees. We give an example in Fig. 13.

Theorem 3.8. The triple $\left(\mathcal{T}_{\bullet}, \bar{*}, \hat{\delta}\right)$ is a graded connected cocommutative Hopf algebra. The triple $\left(\mathcal{T}_{\bullet}, \hat{*}, \bar{\delta}\right)$ is a graded connected Hopf algebra and isomorphic to Springer 


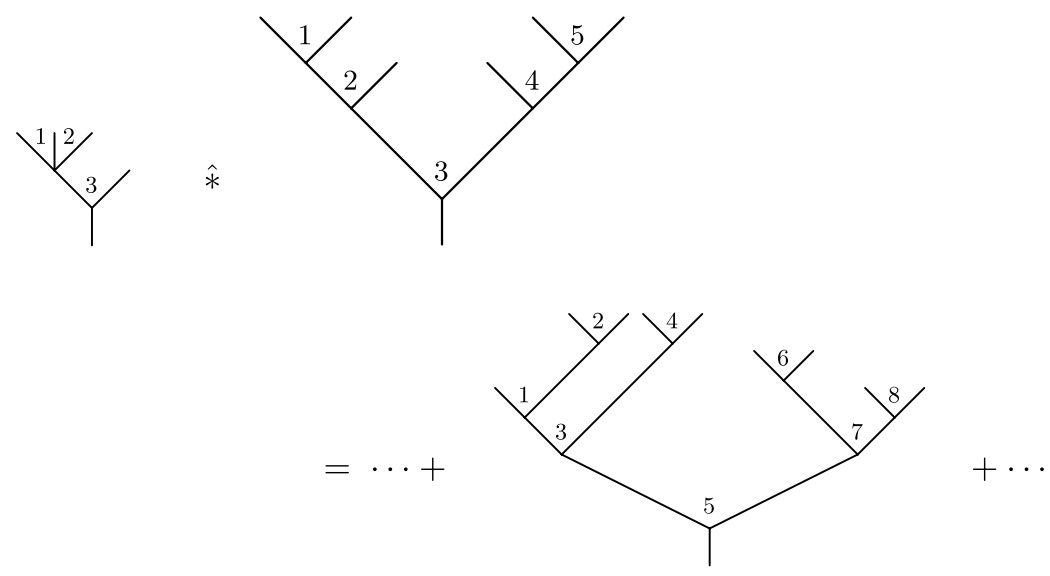

Fig. 13 The $(\{2,4,6\},\{1,3,5,7,8\})$ summand of the symmetrized product $\hat{*}$

the graded dual of the Hopf algebra of quasi-symmetric functions in non-commuting variables, NCQsym*, considered in [3].

This follows from [15, Section 3] and the remarks after Lemmas 3.4 and 3.7.

\section{Freeness and enveloping algebra}

In this section, we study the algebra structures on $\mathcal{T}_{\bullet}$ given by the reduced product $\bar{*}$ and the symmetrized product $\hat{*}$. Both algebras turn out to be free, and we will specify a set of free generators. With an eye on the Cartier-Milnor-Moore theorem [11] (see [14] for a modern combinatorial proof), we will then show that the cocommutative Hopf algebra $\left(\mathcal{T}_{\bullet}, \bar{*}, \hat{\delta}\right)$ is in fact the enveloping algebra of a free Lie algebra.

Let $n \in \mathbb{N}_{0}$ and set Comp ${ }_{n}=\operatorname{Comp}_{[n]}$. We say that a set composition $\left(P_{1}, \ldots, P_{k}\right) \in$ Comp $_{n}$ is reduced if there is no pair $(a, m)$ with $a<k$ and $m<n$ such that $\bigcup_{i=1}^{a} P_{i}=$ $[m]$. This corresponds to the notion of balanced tree, where an increasing tree $T$ with $n$ branchings and $k$ levels is said to be balanced if no pair $(a, m)$ with $a<k$ exists such that the branchings of $T$ with levels in $[a]$ are naturally labeled by the elements of $[m]$. It is clear that each set composition $P$ can be written uniquely as a product $P=P^{(1)} \bar{*} \ldots \bar{*} P^{(m)}$ of reduced set compositions $P^{(1)}, \ldots, P^{(m)}$, where $\bar{*}$ is the restricted product defined in Lemma 3.6. Equivalently, each increasing tree factorizes uniquely as a restricted product of balanced increasing trees. This implies:

Proposition 4.1. $\left(\mathcal{T}_{\bullet}, \bar{*}\right)$ is a free associative algebra, freely generated by the set of reduced set compositions in Comp.

Here we write Comp $=\bigcup_{n \in \mathbb{N}_{0}}$ Comp $_{n}$ for the set of all set compositions of initial subsets of $\mathbb{N}$.

For the next result, we work over the rational number field $\mathbb{Q}$ and consider the vector space $\mathcal{T}_{\bullet}^{\mathbb{Q}}=\mathbb{Q} \otimes_{\mathbb{Z}} \mathcal{T}_{\bullet}$ with $\mathbb{Q}$-basis in bijection with Comp. 
Corollary 4.2. The graded connected cocommutative Hopf algebra $\left(\mathcal{T}_{\bullet}^{\mathbb{Q}}, \bar{*}, \hat{\delta}\right)$ is the enveloping algebra of a free Lie algebra whose set of generators is naturally in bijection with the set of reduced set compositions or, equivalently, of balanced increasing trees.

Proof: This follows from [15, Lemma 22] since all graded components of $\mathcal{T}_{\bullet}^{\mathbb{Q}}$ have finite dimension.

The generators of this free Lie algebra can be computed explicitly, using the techniques of [14] (see also [13]). Let us write Red for the set of reduced set compositions in Comp and $e^{1}$ for the logarithm of the identity of $\mathcal{T}_{\bullet}$ in the convolution algebra of linear endomorphisms of $\mathcal{T}_{\bullet}$. Then the elements $e^{1}(R), R \in \mathrm{Red}$, form a set of free generators for the primitive Lie algebra of $\left(\mathcal{T}_{\bullet}^{\mathbb{Q}}, \bar{*}, \hat{\delta}\right)$. Further details can be found in the proof of [15, Lemma 22].

In concluding this section, we note that Proposition 4.1 holds for the symmetrized algebra $\left(\mathcal{T}_{\bullet}, \hat{*}\right)$ as well. This was shown by Bergeron-Zabrocki [3] and NovelliThibon [12], in the dual setting of quasi-symmetric functions in non-commuting variables. In our approach it follows from Proposition 4.1, by means of a standard triangularity argument: there is a strict total ordering « on set compositions such that, for all $P \in \mathrm{Comp}_{p}, Q \in \mathrm{Comp}_{q}$,

$$
P \hat{*} Q \in P \bar{*} Q+\operatorname{span}_{\mathbb{Z}}\left\{U \in \operatorname{Comp}_{p+q} \mid P \bar{*} Q \ll U\right\} .
$$

We give the details below, for the sake of completeness.

The order $\ll$ is defined as follows. Consider the set $\overline{\mathbb{N}}$ of the positive integers together with the comma symbol: $\overline{\mathbb{N}}:=\mathbb{N} \cup\{$,$\} . We extend the natural order on \mathbb{N}$ to $\overline{\mathbb{N}}$ by putting, $<1$. Any set composition of $[n]$ can be viewed as a word over the alphabet $\overline{\mathbb{N}}$. For example, the set composition $(145,26,3)$ translates into the word $145,26,3$.

If $A, B \subseteq \mathbb{N}, P$ is a set composition of $A$ and $Q$ is a set composition of $B$, we set

$$
P \ll Q
$$

if $|A|<|B|$, or if $|A|=|B|$ and $P$ is smaller than $Q$ with respect to the lexicographic order on words over the alphabet $\overline{\mathbb{N}}$. For example, we have $(245,169) \ll(245,178)$ because $6<7$, and $(23,4569) \ll(234,1,9,5)$ because, $<4$.

We claim that (1) holds. We start with following lemma which is clear from the definition.

Lemma 4.3. Suppose $P, Q, R, S$ are set compositions of $A, B, C, D$, respectively, and that $A \cap C=\emptyset=B \cap D$. If $|A|=|B|$, then $P \ll Q$ implies that $P * R \ll Q * S$.

To see (1) now, note first that $P \hat{*} Q-P \bar{*} Q$ is equal to the sum of all set compositions

$$
U=\operatorname{is}_{[p], A}(P) * \operatorname{is}_{[q], B}(Q),
$$


where $A, B$ are such that $A \amalg B=[p+q],|A|=p$ and $A \neq[p]$. Hence, by Lemma 4.3 , it suffices to show that

$$
P \ll \text { is }_{[p], A}(P)
$$

for all subsets $A$ of $[p+q]$ of order $p$ with $A \neq[p]$. Suppose $P=\left(P_{1}, \ldots, P_{k}\right)$, and let $\iota:[p] \rightarrow A$ be the order-preserving bijection. Then is $[p], A(P)=\left(\iota\left(P_{1}\right), \ldots, \iota\left(P_{k}\right)\right)$. Hence, if $i \in[k]$ is minimal with $P_{i} \neq \iota\left(P_{i}\right)$ and $j \in P_{i}$ is minimal with $j \neq \iota(j)$, then $j<\iota(j)$ because $\iota$ preserves the orders on $[p]$ and $A$. This implies (2), hence also (1).

Theorem 4.4. $\left(\mathcal{T}_{\bullet}, \hat{*}\right)$ is a free associative algebra, freely generated by the set of reduced set compositions in Comp.

Proof: We need to show that the symmetrized products $P^{(1)} \hat{*} \cdots \hat{*} P^{(m)}$ of reduced set compositions $P^{(1)}, \ldots, P^{(m)}$ in Comp form a $\mathbb{Z}$-basis of $\mathcal{T}_{\bullet}$. From Lemma 4.3 and (1) it follows that such a product is contained in

$$
P^{(1)} \bar{*} \cdots \bar{*} P^{(m)}+\operatorname{span}_{\mathbb{Z}}\left\{U \in \operatorname{Comp} \mid P^{(1)} \bar{*} \cdots \bar{*} P^{(m)} \ll U\right\} .
$$

Hence the claim follows from Proposition 4.1, because the reduced products $P^{(1)} \bar{*} \cdots \bar{*} P^{(m)}$ form a $\mathbb{Z}$-basis of $\mathcal{T}_{\bullet}$.

\section{Enveloping algebras and trees}

In this section we consider a number of subspaces of the twisted descent algebra (up to natural identifications), such as the direct $\operatorname{sum} \mathcal{S}_{\bullet}:=\bigoplus_{n \in \mathbb{N}_{0}} \mathbb{Z}\left[S_{n}\right]$ of the symmetric group algebras or the linear span of (planar rooted) trees. We will show how the Hopf algebra structures on set compositions and increasing trees studied in the previous sections restrict to these subspaces. In this way, we will obtain various algebraic structures. Some of them are known: the Malvenuto-Reutenauer Hopf algebra of permutations, and the enveloping algebra structure on $\mathcal{S}_{\bullet}$ introduced in [15]; others seem to be new. As far as we can say, there is no obvious connection between our Hopf algebras of trees and the Hopf algebra structures on trees and forests appearing in renormalization theory (see, for example, $[4,6,7]$ ).

First of all, we show that the enveloping algebra with underlying graded vector space $\mathcal{S}_{\bullet}$, as defined in [15], is a sub-enveloping algebra of the enveloping algebra of twisted descents $\left(\mathcal{T}_{\bullet}, \bar{*}, \hat{\delta}\right)$ considered in Corollary 4.2 (up to an anti-involution). Second, the Malvenuto-Reutenauer Hopf algebra is a Hopf subalgebra of the (non-cocommutative) Hopf algebra of twisted descents $\left(\mathcal{T}_{\bullet}, \hat{*}, \bar{\delta}\right)$.

Third, we turn to (planar rooted) trees and show that the corresponding graded vector space can be provided with an enveloping algebra structure. It embeds, as an enveloping algebra, into the enveloping algebra of increasing trees.

Finally, we show that a stronger result holds for (planar rooted) binary trees, whose associated sub-enveloping algebra of the enveloping algebra of trees also embeds into the enveloping algebra associated to $\mathcal{S}_{\bullet}$. 


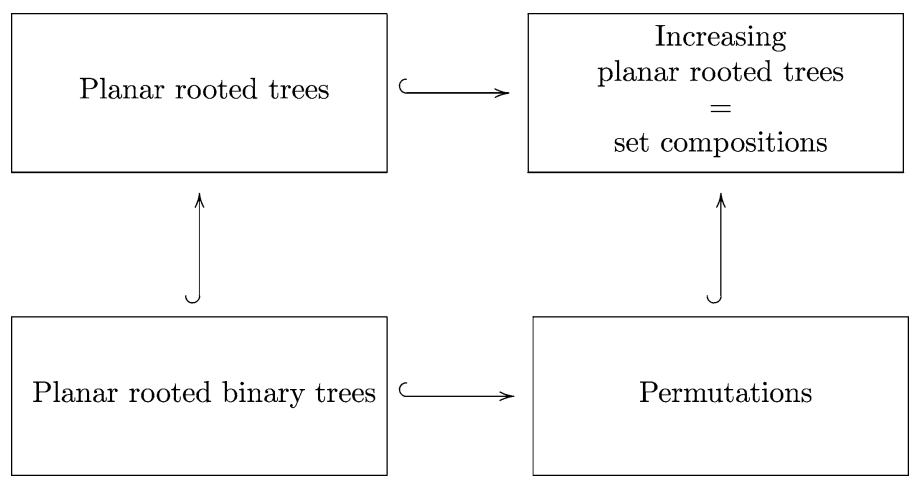

Fig. 14 Diagram of embeddings

These various embeddings of enveloping algebras are induced by embeddings of sets of combinatorial objects (trees, set compositions, and so on) and illustrated in Fig. 14. Note that the embeddings are not canonical; they will be defined below.

As far as the space $\mathcal{S}_{\bullet}$ is concerned, all our results build on the functorial superstructure associated to the corresponding tensor species $\mathcal{S}$. This tensor species is defined on objects by

$$
\mathcal{S}[T]:=\mathbb{Z}\left[\operatorname{Aut}_{\mathrm{Fin}}(T)\right]
$$

for all $T \in$ Fin. If $T=\left\{t_{1}, \ldots, t_{k}\right\} \subseteq \mathbb{N}$ such that $t_{1}<\cdots<t_{k}$, we can think of any bijection $\sigma \in \operatorname{Aut}_{\text {Fin }}(T)$ as the ordered sequence $\left(\sigma\left(t_{1}\right), \ldots, \sigma\left(t_{k}\right)\right)$. The action of a bijection $\phi$ from $T$ to $S$ is then given by

$$
\phi\left(\sigma\left(t_{1}\right), \ldots, \sigma\left(t_{k}\right)\right):=\left(\phi \circ \sigma\left(t_{1}\right), \ldots, \phi \circ \sigma\left(t_{k}\right)\right) .
$$

A twisted Hopf algebra structure on $\mathcal{S}$ can be defined as follows. If $S=$ $\left\{s_{1}, \ldots, s_{k}\right\}, T=\left\{t_{1}, \ldots, t_{l}\right\} \subseteq \mathbb{N}$ such that $S \cap T=\emptyset$ and $\alpha \in \operatorname{Aut}_{\text {Fin }}(S), \beta \in$ $\operatorname{Aut}_{\text {Fin }}(T)$, then

$$
\left(\alpha\left(s_{1}\right), \ldots, \alpha\left(s_{k}\right)\right) \times\left(\beta\left(t_{1}\right), \ldots, \beta\left(t_{l}\right)\right):=\left(\alpha\left(s_{1}\right), \ldots, \alpha\left(s_{k}\right), \beta\left(t_{1}\right), \ldots, \beta\left(t_{l}\right)\right) .
$$

Furthermore, if $\sigma \in \operatorname{Aut}_{\text {Fin }}(S \bigsqcup T)$, then

$$
\delta_{S, T}(\sigma):=\left.\left.\sigma\right|_{S} \otimes \sigma\right|_{T}
$$

Here we write $\left.\sigma\right|_{S}$ for the subsequence of $\sigma$ associated to the elements of $S$. For example, $\left.(3,5,2,4,1)\right|_{\{1,3,5\}}=(3,5,1)$.

The following lemma is a direct consequence of the definitions. 
Lemma 5.1. The canonical embeddings of the symmetric groups $S_{n}$ into Comp , $_{n}$ defined by

$$
\sigma \longmapsto(\sigma(1), \ldots, \sigma(n))
$$

for all $n \in \mathbb{N}_{0}$ and $\sigma \in S_{n}$, yield an embedding of the twisted Hopf algebra $\mathcal{S}$ into the twisted Hopf algebra $\mathcal{T}$ of set compositions.

Recall that to each twisted Hopf algebra is associated a symmetrized Hopf algebra and a cosymmetrized Hopf algebra. The latter is an enveloping algebra (that is, a graded connected cocommutative Hopf algebra) if the twisted Hopf algebra is cocommutative. Since these constructions are natural in a functorial sense, an embedding of twisted Hopf algebras induces embeddings of the associated symmetrized and cosymmetrized Hopf algebras.

The cosymmetrized Hopf algebra stucture on $\mathcal{S}_{\bullet}$ associated with the twisted Hopf algebra $\mathcal{S}$ is defined as follows.

Definition 5.2. The graded vector space $\mathcal{S}_{\bullet}$ is an enveloping algebra with respect to the usual concatenation product $\times$ and the cosymmetrized coproduct $\hat{\delta}$. These are defined by

$$
(\alpha \times \beta)(i):= \begin{cases}\alpha(i), & \text { if } i \leq n, \\ n+\beta(i-n), & \text { if } i>n,\end{cases}
$$

for all $n, m \in \mathbb{N}_{0}, \alpha \in S_{n}, \beta \in S_{m}, i \in[n+m]$, and

$$
\hat{\delta}(\alpha):=\sum_{S \bigsqcup T=[n]} \text { is }_{S}\left(\left.\alpha\right|_{S}\right) \otimes \text { is }_{T}\left(\left.\alpha\right|_{T}\right)
$$

for all $n \in \mathbb{N}_{0}, \alpha \in S_{n}$.

Here is ind $_{S}$ and ${ }_{T}$ are the standardization maps considered earlier so that, for example, is $_{\{1,3,5\}}\left(\left.(3,5,2,4,1)\right|_{\{1,3,5\}}\right)=(2,3,1)$.

A detailed description of the Lie algebra of primitive elements associated with this enveloping algebra is given in [15].

The symmetrized Hopf algebra stucture on $\mathcal{S}_{\bullet}$ associated with the twisted Hopf algebra $\mathcal{S}$ yields the Malvenuto-Reutenauer algebra. We recall its definition.

Definition 5.3. The Malvenuto-Reutenauer algebra $\mathcal{S}_{\bullet}$ is a graded connected Hopf algebra with respect to the convolution product $*$ and the restricted coproduct $\bar{\delta}$. These are defined by

$$
\alpha * \beta:=q_{(n, m)} \cdot(\alpha \times \beta)
$$


for all $n, m \in \mathbb{N}_{0}, \alpha \in S_{n}, \beta \in S_{m}$, and

$$
\bar{\delta}(\alpha):=\left.\sum_{i=0}^{n} \alpha\right|_{[i]} \otimes \mathrm{is}_{\{i+1, \ldots, n\}}\left(\left.\alpha\right|_{\{i+1, \ldots, n\}}\right)
$$

for all $n \in \mathbb{N}_{0}, \alpha \in S_{n}$.

Here we write $q_{(n, m)}$ for the sum in $\mathbb{Z}\left[S_{n+m}\right]$ of all permutations $\pi \in S_{n+m}$ such that $\pi(1)<\cdots<\pi(n)$ and $\pi(n+1)<\cdots<\pi(n+m)$.

Note that these definitions agree with the structures studied in [15] only up to the involution inv $: \mathcal{S}_{\bullet} \rightarrow \mathcal{S}_{\bullet}$ which maps any permutation to its inverse; for, in that article, twisted bialgebras were studied from Barratt's point of view [1] (that is, by considering right modules over symmetric groups or, equivalently, by considering contravariant functors from the category of finite sets and bijections), whereas here and in [16], twisted bialgebras have been studied from Joyal's point of view [8] (that is, by considering left modules over symmetric groups or, equivalently, by considering covariant functors). As usual, one can move from one point of view to the other using the map inv. Details on the two point of views and their relative behaviours can be found in the first section of [15].

From Lemma 5.1, we can now deduce without further ado:

Theorem 5.4. The canonical embedding of $\mathcal{S}_{\bullet}$ into $\mathcal{T}_{\bullet}$ given by (3) is an embedding of enveloping algebras $\left(\mathcal{S}_{\bullet}, \bar{*}, \hat{\delta}\right) \rightarrow\left(\mathcal{T}_{\bullet}, \bar{*}, \hat{\delta}\right)$. It is also an embedding of the MalvenutoReutenauer Hopf algebra into $\left(\mathcal{T}_{\bullet}, \hat{*}, \bar{\delta}\right)$.

We now turn to the enveloping algebra structures on trees and binary trees and consider $\mathcal{T}_{\bullet}$ as the linear span of all increasing trees. Recall that the product of two increasing trees in the twisted algebra of increasing trees is the grafting of the first tree on the left most leaf of the second. If we assume, for simplicity, that the first tree has levels $1, \ldots, n$ and the second tree has non-standard levels $n+1, \ldots, n+m$, then the level of each branching in the product is the same before and after the grafting.

The same operation (grafting on the left most leaf) defines an associative product on the linear span of (planar rooted) trees. The forgetful map

$$
\text { Fgt }: \mathcal{T}_{\bullet} \rightarrow \overline{\mathcal{T}}_{\bullet}
$$

from increasing trees to trees is clearly an algebra map, where we write

$$
\overline{\mathcal{T}}_{\bullet}=\bigoplus_{n \in \mathbb{N}_{0}} \overline{\mathcal{T}}_{n}
$$

for the graded vector space with basis the set of trees, graded by the number of branchings.

We claim that Fgt has a section Inc in the category of graded associative algebras with identity. Let $T$ be any non-empty planar rooted tree with $n$ branchings. Then $T$ can be written uniquely as a wedge $T=\bigvee\left(T_{0}, \ldots, T_{m}\right)$. Let $b_{1}, \ldots, b_{m}$ denote the natural labels of the root branchings of $T$. We assume inductively that an increasing 悬Springer 
tree $\left(T_{i}, \lambda_{i}\right)=\operatorname{Inc}\left(T_{i}\right)$ has been defined for all $0 \leq i \leq m$. A level function $\lambda$ on $T$ can then be defined by requiring that:

(i) $\left(T_{i}, \lambda_{i}\right)$ is the $S_{i}$-contraction of $(T, \lambda)$ for all $0 \leq i \leq m$, where $S_{i}$ is the set of branchings of $T$ which belong to $T_{i}$ (embedded in $T$ ).

(ii) The levels on $T_{i}$ (embedded in $T$ ) are strictly less than the levels on $T_{j}$ (embedded in $T$ ), for all $0 \leq i<j \leq m$.

We set $\operatorname{lnc}(T):=(T, \lambda)$ and observe:

Lemma 5.5. The map Inc from trees to increasing trees is a section of the forgetful map Fgt. It defines an embedding of algebras $\left(\overline{\mathcal{T}}_{\bullet}, \bar{*}\right) \rightarrow\left(\mathcal{T}_{\bullet}, \bar{*}\right)$ where $\bar{*}$ is the left grafting product on $\overline{\mathcal{T}}_{\bullet}$ (by slight abuse of notation) and the restricted product on $\mathcal{T}_{\bullet}$.

The proof is geometrically straightforward and left to the reader.

Any increasing tree in the image of Inc is called left increasing. Due to the recursive definition of Inc, left increasing trees can be characterized as follows.

Lemma 5.6. Let $(T, \lambda)$ be an increasing tree. Then $(T, \lambda)$ is left increasing if and only if, for any branchings $b$ and $b^{\prime}$ of $T$, we have $\lambda(b)<\lambda\left(b^{\prime}\right)$ whenever $b$ is to the left of $b^{\prime}$ in $T$ and the associated vertices $v, v^{\prime}$ do not lie on a common path connecting $a$ leaf of $T$ with the root of $T$.

Corollary 5.7. Any contraction of a left increasing tree is left increasing.

Proof: It is enough to understand how all three notions occuring in Lemma 5.6 (to lie on a common path, to lie further to the left, to have a smaller level) behave with respect to the contraction process.

Let $\left(T^{\prime}, \lambda^{\prime}\right)$ be a contraction of a left increasing tree $(T, \lambda)$. Let $b, b^{\prime}$ be branchings of $T^{\prime}$, and denote the corresponding branchings of $T$ by $\tilde{b}$ and $\tilde{b}^{\prime}$, respectively.

The contraction process is an order preserving map with respect to the natural labelling of branchings. This follows directly from its recursive left-to-right definition. In particular, $b$ is to the left of $b^{\prime}$ in $T^{\prime}$ if and only if $\tilde{b}$ is to the left of $\tilde{b}^{\prime}$ in $T$.

Furthermore, we have $\lambda^{\prime}(b)<\lambda^{\prime}\left(b^{\prime}\right)$ if and only if $\lambda(\tilde{b})<\lambda\left(\tilde{b}^{\prime}\right)$ since $\lambda^{\prime}$ is the standardization of the restriction of $\lambda$ to the set of branchings of $T^{\prime}$.

Let us assume that $b$ is to the left of $b^{\prime}$. As mentioned in Section 2, the vertices associated to $\tilde{b}$ and $\tilde{b}^{\prime}$ lie on a common path from a leaf to the root of $T$ if and only if either $\lambda(\tilde{b})<\lambda\left(\tilde{b}^{\prime}\right)$ and for any branching $k$ between $\tilde{b}$ and $\tilde{b}^{\prime}$ in the left-toright ordering $\lambda(k)>\lambda(\tilde{b})$, or $\lambda(\tilde{b})>\lambda\left(\tilde{b}^{\prime}\right)$ and, with the same notation, $\lambda(k)>\lambda\left(\tilde{b}^{\prime}\right)$. Since the same characterization holds for $b$ and $b^{\prime}$ in $T^{\prime}$, and since levels (up to standardization) and the left-to-right ordering are preserved by the contraction process, it follows that the property of lying on a common path from a leaf to the root is preserved by the contraction $T \mapsto T^{\prime}$.

In particular, if $b$ and $b^{\prime}$ do not lie on a common path, the same property is true for $\tilde{b}$ and $\tilde{b}^{\prime}$. Since $T$ is left increasing, we get $\lambda(\tilde{b})<\lambda\left(\tilde{b}^{\prime}\right)$ and $\lambda(b)<\lambda\left(b^{\prime}\right)$, which concludes the proof. 
As a consequence of the preceding result, the cosymmetrized coproduct $\hat{\delta}$ restricts to a coproduct on the $\mathbb{Z}$-linear span of left increasing trees, $\operatorname{Inc}\left(\overline{\mathcal{T}}_{\bullet}\right)$, in $\mathcal{T}_{\text {. }}$. Combined with Lemma 5.5, this gives:

Theorem 5.8. The linear span of left increasing trees (or, equivalently, the linear span of trees) is a Hopf subalgebra of the cocommutative Hopf algebra of increasing trees. In particular, this Hopf algebra of trees is an enveloping algebra. It is free as an algebra, and the enveloping algebra of a free Lie algebra.

To conclude, we observe that the left increasing tree $\operatorname{Inc}(T)$ corresponding to a binary tree $T$ is characterized by the property that it has a single grafting at each level. This property is also preserved by the contraction process. Furthermore, the set composition $(\sigma(1), \ldots, \sigma(n))$ corresponding to a permutation $\sigma \in S_{n}$ can be characterized in the same way. Hence we get from Theorem 5.8:

Corollary 5.9. The linear span of (planar rooted) binary trees is naturally embedded in $\mathcal{S}_{\bullet}$ and $\mathcal{T}_{\bullet}$ as an enveloping algebra, and is the enveloping algebra of a free Lie algebra.

As far as we can say, there is no direct connection between our Hopf algebra of planar binary trees and Loday-Ronco's [10].

\section{References}

1. M.G. Barratt, "Twisted Lie algebras. Geometric applications of homotopy theory," in Proceedings of the Conference, Evanston 1977. Lecture Notes in Mathematics 658, Springer, Berlin, 1978, pp. 9-15.

2. N. Bergeron, C. Reutenauer, M. Rosas, and M. Zabrocki, "Invariants and Coinvariants of the Symmetric Group in Noncommuting Variables," Preprint arXiv:math.RA/0502082.

3. N. Bergeron and M. Zabrocki, "The Hopf algebras of symmetric functions and quasisymmetric functions in non-commutative variables are free and cofree," Preprint arXiv:math.RA/0505137.

4. C. Brouder and A. Frabetti, "QED Hopf algebras on planar binary trees," J. Alg. 267(1) (2003), $298-322$.

5. F. Chapoton, "Algèbres de Hopf des permutahèdres, associahèdres et hypercubes," Adv. Math. 150(2), (2000), 264-275.

6. A. Connes and D. Kreimer, "Hopf algebras, renormalization and noncommutative geometry," Commun. Math. Phys. 199(1) (1998), 203-242.

7. H. Figueroa and J. Gracia-Bondia, "Combinatorial Hopf algebras in quantum field theory I," Preprint arXiv:hep-th/0408145.

8. A. Joyal, "Foncteurs analytiques et espèces de structures," in Combinatoire énumérative, Proc. Colloq., Montréal, Canada, 1985. Lecture Notes in Mathematics 1234, Springer, Berlin, 1986, pp. 126-159.

9. J.-L. Loday, "Dialgebras," in: Dialgebras and Related Operads," Lecture Notes in Mathematics 1736, Springer, Berlin, 2001, pp. 7-66.

10. J.-L. Loday and M.O. Ronco, "Hopf algebra of the planar binary trees," Adv. Math. 139(2) (1998), 293-309.

11. J.W. Milnor and J.C. Moore, "On the structure of Hopf algebras," Ann. of Math. 81(2) (1965), 211-264.

12. J.-C. Novelli and J.-Y. Thibon, "Polynomial realizations of some trialgebras," Preprint arXiv:math.CO/0605061.

13. F. Patras, "La décomposition en poids des algèbres de Hopf," Ann. Inst. Fourier (Grenoble) 43(4) (1993), 1067-1087.

14. F. Patras, "L'algèbre des descentes d'une bigèbre graduée," J. Alg. 170(2) (1994), 547-566.

15. F. Patras and C. Reutenauer, "On descent algebras and twisted bialgebras," Moscow Math. J. 4(1) (2004), 199-216. 
16. F. Patras and M. Schocker, "Twisted descent algebras and the Solomon-Tits algebra," Adv. in Math. 199(1) (2006), 151-184.

17. D. Rawlings, "The ABC's of classical enumeration," Ann. Sci. Math. Québec. 10(2) (1986), 207-235.

18. M. Schocker, "The module structure of the Solomon-Tits algebra of the symmetric group," J. Alg. 301(2) (2006), 554-586.

19. R. Stanley, Enumerative combinatorics, Volume I, The Wadsworth and Brooks/Cole Mathematics Series. Monterey, California, 1986.

20. C.R. Stover, "The equivalence of certain categories of twisted Lie and Hopf algebras over a commutative ring," J. Pure Appl. Algebra 86(3) (1993), 289-326.

21. A. Tonks, "Relating the associahedron and the permutohedron," Operads: Proceedings of Renaissance Conferences (Hartford, CT/Luminy, 1995), 33-36, Contemp. Math., 202, Amer. Math. Soc., Providence, RI, 1997. 